\section{Grouped Vesicular Lesions in an Infant}

An 8-month-old female infant presented with vesicular lesions on the right lower extremity for 2 days. There were grouped vesicles on an erythematous base over right leg, dorsum of right foot and sole, distributed in the L5 and S1 dermatomes (Fig 1). The baby was irritable, but afebrile. The baby was born at term and by normal vaginal delivery and her birth weight was $2.5 \mathrm{~kg}$. Postnatal period was uneventful. Her developmental milestones were within normal range. There was history of maternal varicella infection during 3rd months of pregnancy. Tzanck smear from the lesions showed mononuclear and multinucleated acantholytic cells with ground glass nuclei, consistent with Tzanck cells. Based on history and clinical findings, diagnosis of Herpes zoster was made. The infant was treated symptomatically with topical calamine lotion and oral antipyretic. The lesions crusted in 1 week and resolved completely in 2 weeks, without any sequelae.

Herpes Zoster (HZ) results from reactivation of varicella Zoster virus (VZV) that entered the cutaneous nerves during an earlier episode of chickenpox, traveled to the dorsal root ganglia, and remained in a latent form. Age, immunosuppressive drugs, lymphoma, fatigue, emotional upsets, and radiation therapy have been implicated in reactivation of the virus, which subsequently travels back down the sensory nerve, infecting the skin. Reactivation of latent VZV infection is very rare in childhood, more so in infants. Infantile $\mathrm{HZ}$ is more commonly associated with intrauterine $\mathrm{VZV}$ infection than postnatal infection. $\mathrm{HZ}$ in children is considered common in immunocompromised babies, but can occur in immunocompetent children as well. The diagnosis can usually be made on clinical grounds. Tzanck smear may support the clinical diagnosis. The differential diagnoses are zosteriform herpes simplex infection (no radiating pain, small vesicles of almost uniform size, less number of groups of vesicles, and more

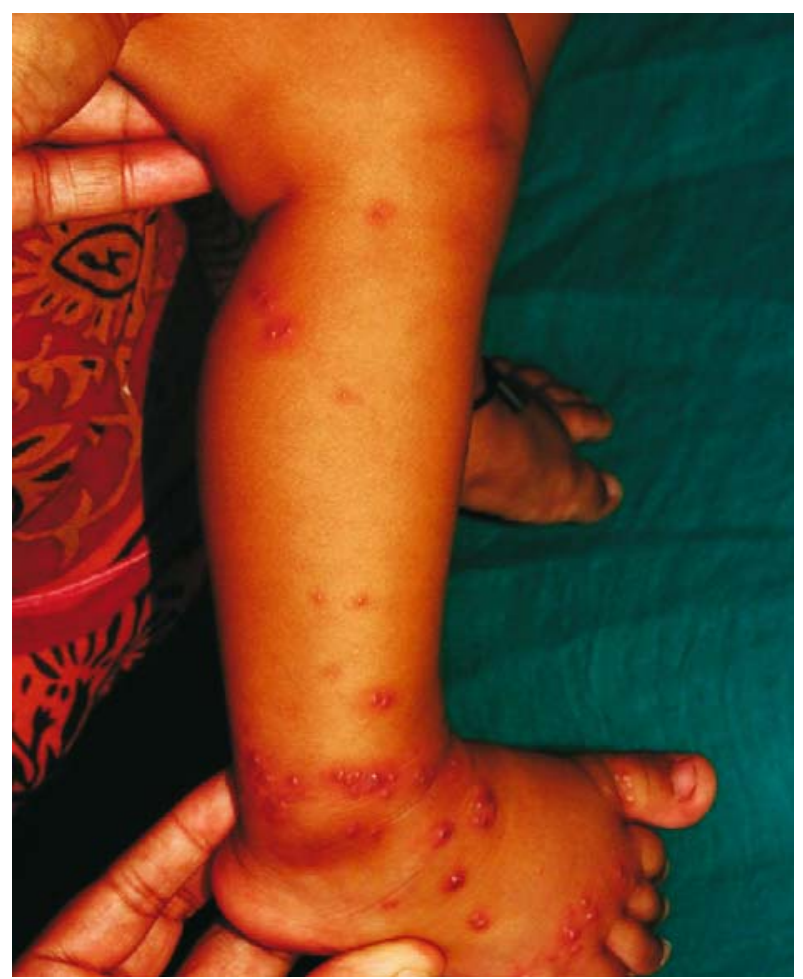

FIG 1 Grouped vesicles on an erythematous base, present unilaterally on right lower extremity. Note central umbilication in some of the vesicles.

likely to recur) and contact dermatitis (history of contact, and presence of papules, pustules, scaling and/ or epidermal necrosis). HZ is a self-limited cutaneous eruption in children and treatment usually consists of supportive care with antihistamines and antipruritic agents. Systemic antiviral drug is usually recommended in severe disseminated VZV infection, ophthalmic VZV infection and in immunocompromised patients.

\footnotetext{
AviJit Mondal, Neloy Sinha And *Piyush Kumar Department of Dermatology, College of Medicine and JNM Hospital; and * Katihar Medical College and Hospital Karim Bagh, Katihar - 854105 Bihar, India. docpiyush@gmail.com
} 